\title{
Factors contributing to nonadherence to follow-up appointments in a resident glaucoma clinic versus primary eye care clinic
}

This article was published in the following Dove Press journal:

Patient Preference and Adherence

8 January 2016

Number of times this article has been viewed

\section{Scott J Fudemberg' \\ Brian Lee' \\ Michael Waisbourd' \\ Rachel A Murphy' \\ Yang Dai' \\ Benjamin E Leiby ${ }^{2}$ \\ Lisa A Hark'}

'Glaucoma Research Center, Wills Eye Hospital, Philadelphia, PA, USA; ${ }^{2}$ Department of Pharmacology and Experimental Therapeutics, Thomas Jefferson University, Philadelphia, PA, USA
Correspondence: Scott J Fudemberg Glaucoma Research Center, Wills Eye Hospital, 840 Walnut Street, Suite I I40, Philadelphia, PA 19107, USA

$\mathrm{Tel}+\mathrm{I} 2159283197$

Fax + I 2159283285

Email sjf003@gmail.com
Purpose: To determine the rate of adherence to follow-up appointment recommendations in a resident glaucoma clinic with no mechanism for reminders, compared to a resident cataract and primary eye care (CPEC) clinic in which telephone reminders were used, and to identify factors that contribute to adherence in each patient group.

Methods: This retrospective cohort study included subjects in the CPEC clinic who received telephone reminders and those in the glaucoma clinic who did not. Each sample was selected to have a similar proportion of follow-up recommendations for 1, 3, and 6 months. Subjects were considered adherent if they returned within a specified timeframe.

Results: A total of 144 subjects from the glaucoma clinic and 151 subjects from the CPEC clinic were included. There was no significant difference between follow-up adherence rates of patients who received telephone reminders and those who did not (odds ratio $[\mathrm{OR}]=1.35$, 95\% confidence interval $[\mathrm{CI}] 0.79-2.32, P=0.28$ ). Patients who were on more than two ocular medications were more likely to return for follow-up $(\mathrm{OR}=3.11,95 \% \mathrm{CI} 1.53-6.35, P=0.0018)$. Subjects between the ages 50 and 80 years were more likely to be adherent compared to their younger and older peers $(P=0.02)$.

Conclusion: The follow-up adherence of patients in a CPEC clinic who received telephone reminders was similar to patients in a glaucoma clinic who did not receive any intervention to increase their adherence. Younger ( $<50$ years old) and elderly ( $>80$ years old) subjects, as well as patients using less than two glaucoma medications, were less likely to adhere to their follow-up appointments.

Keywords: glaucoma, retrospective studies, patient adherence, telephone reminders, age, medications

\section{Introduction}

Glaucoma, a chronic progressive degeneration of the optic nerve, is among the most common causes of irreversible blindness worldwide. ${ }^{1,2}$ Patients are often asymptomatic in early stages, but many develop significant and irreversible vision loss as glaucoma progresses. Consistent monitoring of glaucoma progression is essential in reducing intraocular pressure (IOP) and preserving vision.

The American Academy of Ophthalmology recommends that all patients with glaucoma be seen by an ophthalmologist at least every 6-12 months for a comprehensive eye examination. Patients with progressive damage related to glaucoma require close follow-up every 1-2 months, while those with elevated IOP are recommended follow-up every 3-6 months. ${ }^{3}$ Follow-up adherence is important for glaucoma control because it is associated with markers for early disease and improved prognosis, especially since effective therapy is available. ${ }^{4-6}$ Adhering to follow-up recommendations allows the physician to better track glaucoma progression and helps decrease the occurrence of adverse events. 
Complying with follow-up recommendations is also associated with increased medication adherence, which is important for slowing glaucoma progression. ${ }^{7,8}$ Shorter follow-up intervals have been shown to be related to increased adherence to ocular medication, ${ }^{9}$ while patients with longer follow-up intervals are more likely to skip ocular medication doses. ${ }^{10}$

Various studies have found that only about half of patients with glaucoma are adherent to their ophthalmologist's follow-up recommendations, and many patients become lost to follow-up. ${ }^{5,11,12}$ Social and demographic factors may contribute to poor adherence; patients who are young, male, African American or Latino, or who live far from their eye care provider are at a greater risk of nonadherence to follow-up appointments. ${ }^{12,13}$ Furthermore, those who have less severe disease and those who are on multiple ocular medications are also less likely to comply with recommendations. ${ }^{12}$

Several risk factors for poor adherence are potentially modifiable. For example, patients with a poor understanding of glaucoma and its treatment are less likely to be adherent to attending follow-up appointments. ${ }^{13}$ However, even patients who understand the risk of blindness may be nonadherent because they discount the seriousness of their disease, are asymptomatic, are unwilling to pay for eye examinations, or do not know when to return for follow-up. ${ }^{5}$

Little is known about follow-up adherence rates for glaucoma treatment in resident-run eye care clinics. In general, resident clinics may provide care to underserved and minority populations. In addition to socioeconomic challenges, these groups often have high rates of disease and poor adherence. ${ }^{14,15}$ Thus, these differences result in significant disparities in health care outcomes when compared to private practice clinics, such as in perceived quality and continuity of care. ${ }^{16}$

Stubbs et $\mathrm{al}^{17}$ reviewed various methods of appointment reminders and found that telephone reminders are most effective in reducing no show rates. Their meta-analysis found that telephone reminders reduced nonattendance by $9.4 \%$, text messaging by $8.6 \%$, and letters by $7.6 \% .{ }^{17}$ At the Wills Eye Hospital, the cataract and primary eye care (CPEC) service uses telephone appointment reminders, while the resident glaucoma clinic does not use any method to remind patients of their appointments. Both clinics are managed by residents and supervised by attending physicians. The purpose of this study was to compare follow-up adherence rates of patients diagnosed with glaucoma at these two clinics in a high volume, urban eye care setting.

\section{Materials and methods}

This retrospective, cohort study reviewed electronic records of patients who visited the Wills Eye resident glaucoma clinic or CPEC clinic between September 1, 2013 and November 30, 2013.

The electronic medical record was searched for patients with a 365.XX ICD 9 billing code, and patients were included if they were diagnosed as glaucoma or glaucoma suspect, had a documented follow-up recommendation between 1 and 6 months after the index visit, and were $\geq 21$ years of age. Patients were excluded if the chart did not include a recommendation to return for follow-up care in the same clinic. Reasons for no follow-up recommendation included documentation of a terminal illness, relocation, or consultation for a second opinion.

Baseline demographics and clinical characteristics were determined from the initial visit. These included age, gender, race, insurance type, insurance copayment amount, glaucoma status, ocular medications, new patient status, history of prior glaucoma surgery, visual acuity, IOP, and cup-to-disc ratio (CDR). Visual field mean deviation (MD) results within 6 months of the initial visit were also obtained if available.

Of the subjects meeting inclusion criteria, 295 patients were randomly selected in this study such that the samples from both clinics had similar follow-up recommendations. Sixty-seven subjects with 1-month, 68 subjects with 3-month, and 16 subjects with 6-month follow-up recommendations for a total of 151 patients from the CPEC clinic were included. All subjects with recommended 6-month follow-up in the glaucoma clinic were included $(n=15)$, in addition to 66 subjects with 1-month and 63 subjects with 3-month follow-up recommendations from the glaucoma clinic were included (Figure 1). Scheduling and appointment reminder procedures of each clinic are shown in Figure 2.

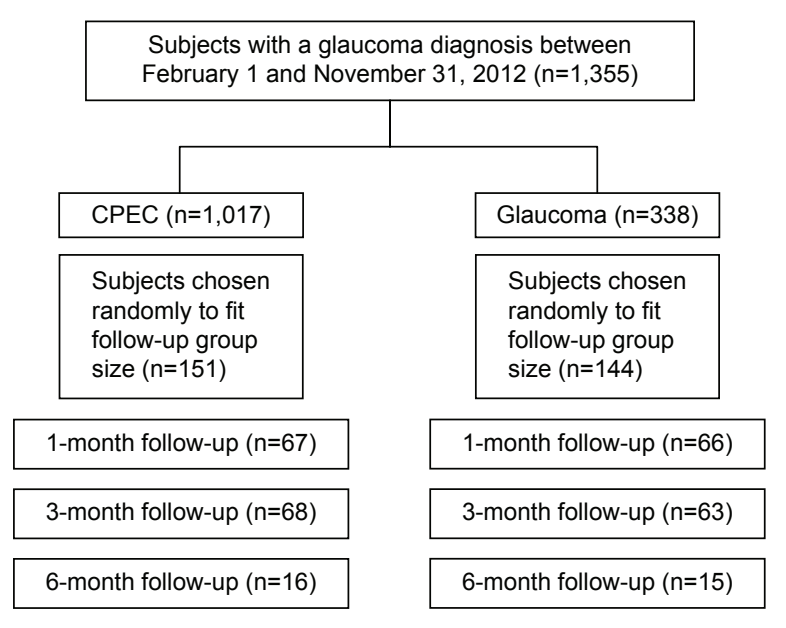

Figure I Procedure for sample selection. Abbreviation: CPEC, cataract and primary eye care. 

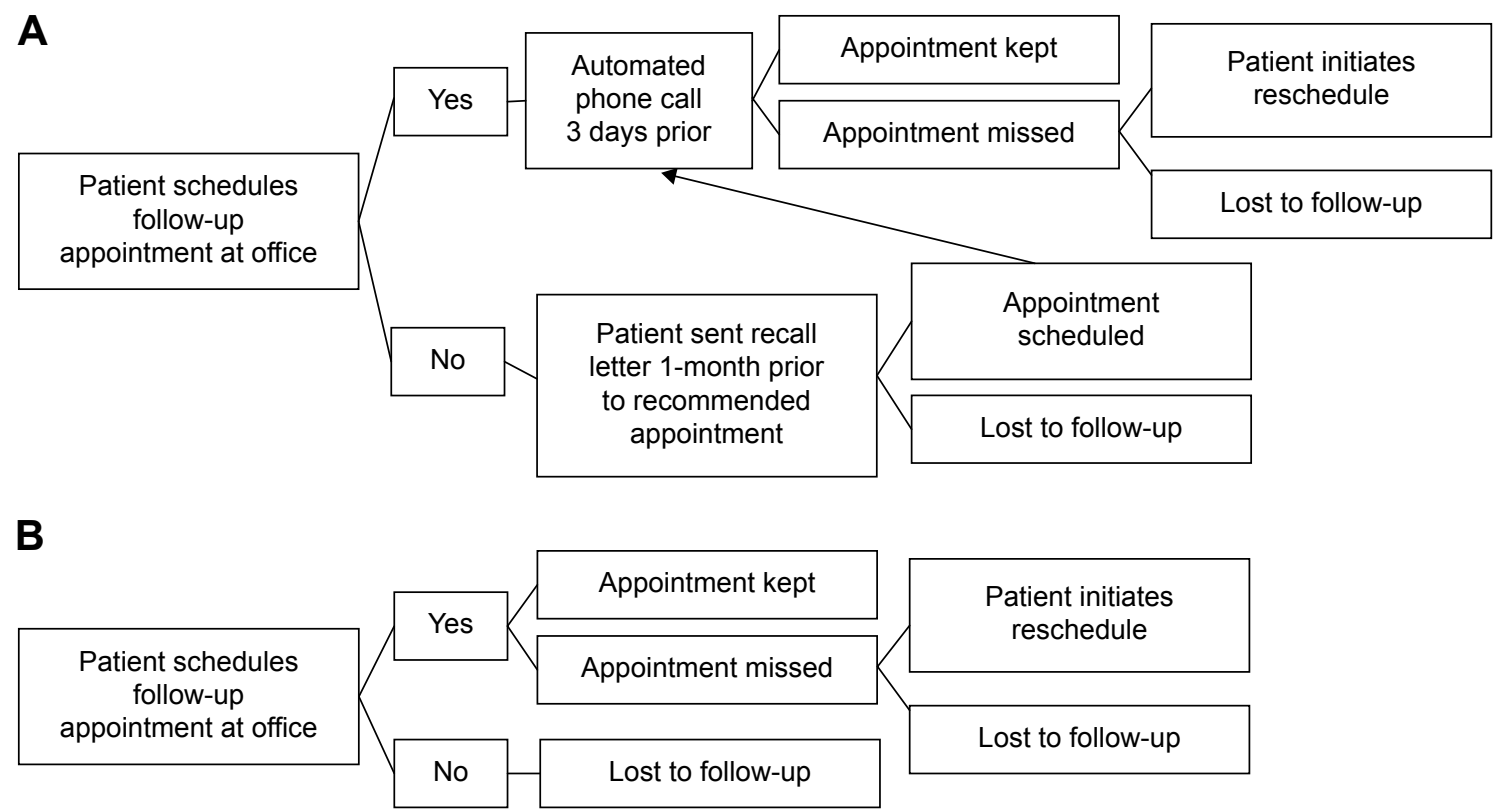

Figure 2 Scheduling procedure for the (A) CPEC clinic and the (B) glaucoma clinic, the control group. Abbreviation: CPEC, cataract and primary eye care.

\section{CPEC clinic appointment procedure (phone group)}

At the CPEC clinic, patients scheduled an appointment at the end of their visit or were sent a recall letter 1-month prior to their recommended follow-up appointment. If they scheduled an appointment, they received an automated phone call reminder 3 days prior to their appointment.

\section{Resident glaucoma clinic appointment procedure (control group)}

At the resident glaucoma clinic, patients scheduled an appointment at the end of their office visit. If they missed their appointment and did not reschedule, they were considered lost to follow-up.

\section{Adherence criteria}

Patients were considered adherent if they met the following cut-off dates: for a 1-month recommendation, a visit within 6 weeks; for a 3-month recommendation, a visit within 4 months; and for a 6-month recommendation, a visit within 8 months.

\section{Statistical analysis}

The baseline characteristics of the two clinics were compared using chi-squared statistics, as were differences between the adherent and nonadherent patient groups. Our sample size gave an $80 \%$ power to detect a difference in adherence rate of $15 \%$, assuming the lower adherence rate was at least $60 \%$. A multivariable logistic regression model was used to estimate the association of clinic with adherence adjusted for other factors associated with adherence. Any variable associated with adherence at the $P<0.25$ in univariable analysis was included in the regression model. A backward elimination procedure was used to select the final model keeping all variables with $P<0.25$ and forcing clinic into the model. All analyses were performed using SAS 9.4 (SAS Institute, Cary, NC, USA).

\section{Statement of ethics}

We certify that all applicable institutional and governmental regulations concerning the ethical use of human volunteers were followed during this research. The Institutional Review Board of the Wills Eye Hospital approved the study protocol and did not require patient consent be provided, as this was a retrospective study.

\section{Results}

Two hundred and ninety-five subjects were included in this study, 151 in the CPEC clinic and 144 in the glaucoma clinic, the control group. The mean age of patients was 64.4 years in the CPEC clinic and 66.3 years $(P=0.27)$ in the glaucoma clinic. The demographic and clinical characteristics of the subjects from each clinic are listed in Table 1. Subjects in the glaucoma clinic had lower copays $(P<0.0001)$, and were more likely to have had no insurance $(P=0.05)$, definite glaucoma diagnoses as opposed to glaucoma suspect diagnoses $(P<0.0001)$, been on greater than two ocular medications $(P<0.0001)$, been returning patients $(P=0.0019)$, and had prior glaucoma surgery $(P<0.0001)$. Additionally, glaucoma 
Table I Baseline characteristics of patients in the CPEC $(N=|5|)$ and glaucoma clinics $(\mathrm{N}=\mid 44)$

\begin{tabular}{|c|c|c|c|}
\hline Characteristic & CPEC, n (\%) & Glaucoma, n (\%) & $P$-value \\
\hline \multicolumn{3}{|l|}{ Age (years) } & 0.67 \\
\hline$<50$ & $22(14.6)$ & $16(11.1)$ & \\
\hline $50-80$ & $103(68.2)$ & $103(7 \mid .5)$ & \\
\hline$>80$ & $26(I 7.2)$ & $25(17.4)$ & \\
\hline \multicolumn{3}{|l|}{ Sex } & 0.28 \\
\hline Female & $83(55.0)$ & $70(48.6)$ & \\
\hline Male & $68(45.0)$ & $74(51.4)$ & \\
\hline \multicolumn{3}{|l|}{ Race } & 0.42 \\
\hline Black & $97(65.5)$ & $97(71.3)$ & \\
\hline White & $35(23.7)$ & $25(18.4)$ & \\
\hline Other & $19(12.6)$ & $22(15.3)$ & \\
\hline \multicolumn{3}{|l|}{ Insurance type } & 0.05 \\
\hline None & $8(5.3)$ & $13(9.0)$ & \\
\hline Medicaid & $23(15.2)$ & $22(15.3)$ & \\
\hline Medicare & $62(4 I . I)$ & $74(5 \mid .4)$ & \\
\hline Private & $58(38.4)$ & $35(24.3)$ & \\
\hline \multicolumn{3}{|c|}{ Insurance copayment } & $<0.0001$ \\
\hline 0 & $66(43.7)$ & II I (77.1) & \\
\hline US\$I-\$20 & $21(13.9)$ & $9(6.3)$ & \\
\hline$>$ US $\$ 20$ & $64(42.4)$ & $24(16.7)$ & \\
\hline \multicolumn{3}{|l|}{ Glaucoma status } & $<0.0001$ \\
\hline Suspect & $122(80.8)$ & $29(20.1)$ & \\
\hline Definite & $29(19.2)$ & II 15 (79.9) & \\
\hline \multicolumn{3}{|l|}{ Medications } & $<0.0001$ \\
\hline$\leq 2$ & $133(88.1)$ & $86(59.7)$ & \\
\hline$>2$ & $18(11.9)$ & $58(40.3)$ & \\
\hline \multicolumn{3}{|l|}{ New patient } & 0.003 \\
\hline No & $84(55.6)$ & $104(72.2)$ & \\
\hline Yes & $67(44.4)$ & $40(27.8)$ & \\
\hline \multicolumn{3}{|c|}{ Prior glaucoma surgery } & $<0.0001$ \\
\hline No & 149 (98.7) & $89(61.8)$ & \\
\hline Yes & $2(1.3)$ & $55(38.2)$ & \\
\hline \multicolumn{3}{|l|}{ Visual acuity } & $<0.0001$ \\
\hline $20 / 20-20 / 100$ & $125(82.8)$ & $78(54.2)$ & \\
\hline$>20 / 100$ & $26(17.2)$ & $66(45.8)$ & \\
\hline \multicolumn{3}{|l|}{ IOP (mmHg) } & 0.0024 \\
\hline$<21$ & $129(85.4)$ & $102(70.8)$ & \\
\hline$\geq 21$ & $22(14.6)$ & $42(29.2)$ & \\
\hline \multicolumn{3}{|l|}{ CDR } & $<0.0001$ \\
\hline$<0.8$ & III (85.4) & 39 (37.9) & \\
\hline$\geq 0.8$ & $19(14.6)$ & $64(62.1)$ & \\
\hline \multicolumn{3}{|l|}{ VF MD } & 0.0001 \\
\hline$>-2$ & $35(42.7)$ & $3(7.9)$ & \\
\hline$\leq-2$ & $47(57.3)$ & $35(92.1)$ & \\
\hline \multicolumn{3}{|l|}{ Rec follow-up } & 0.97 \\
\hline I-month & $67(44.4)$ & $66(45.8)$ & \\
\hline 3-month & $68(45.0)$ & $63(43.8)$ & \\
\hline 6-month & $16(10.6)$ & $15(10.4)$ & \\
\hline
\end{tabular}

Abbreviations: CDR, cup-to-disc ratio; CPEC, cataract and primary eye care; IOP, intraocular pressure; Rec, recommended; VF MD, visual field mean deviation.

clinic patients were more likely to have a visual acuity worse than $20 / 100(P<0.0001)$, IOP $\geq 21 \mathrm{mmHg}(P=0.0024)$, $\mathrm{CDR} \geq 0.8(P<0.0001)$, or visual field $\mathrm{MD} \leq-2 \mathrm{~dB}$ $(P=0.0002)$ in at least one eye. There were no significant differences in gender or race between the groups.
We found no significant difference in follow-up rates in the CPEC clinic (65.6\%) compared to the glaucoma clinic (68.1\%) (odds ratio [OR] $=0.8995 \%$, confidence interval [CI] $0.55-1.45, P=0.65)$. The demographic and clinical characteristics of the adherent and nonadherent patients are listed in Table 2. There were no significant differences between adherent and nonadherent patients in gender, race, insurance type, insurance copayment amount, glaucoma status, whether or not it was the first visit for the patient, history of prior glaucoma surgery, visual acuity, IOP, CDR, or visual field MD. Patients younger than 50 years and older than 80 years were less likely to be adherent $(P=0.038)$. Patients who were adherent were significantly more likely to be on more than two ocular medications $(P=0.0005)$.

Variables with a $P$-value $<0.25$ were included in a multivariable logistic regression model. These included: age group, gender, number of ocular medications ( $<2 \mathrm{vs} \geq 2$ ), follow-up recommendation (1-month vs 3 -month vs 6-month), and whether or not it was the first visit for the patient. In addition, since the adherence rates in different service departments are main concern for this study, the variable for service is kept in the model. The ORs of these variables for predicting patient adherence are listed in Table 3. The adjusted OR for service (CPEC vs glaucoma) was 1.35 (95\% CI $0.79-2.32 ; P=0.28$ ) indicating a small, statistically nonsignificant increase in adherence among patients who received phone calls. Patients with two or fewer ocular medications (OR $=0.32,95 \%$ CI $0.16-0.66$ ) were less likely to adhere to follow-up recommendations compared to patients taking more than two medications. Patients older than 80 years were also less likely to adhere to follow-up compared to those aged $50-80$ years (OR $=0.45,95 \%$ CI $0.23-0.87)$; patients younger than 50 years were marginally less likely to adhere to follow-up as well $(\mathrm{OR}=0.47,95 \% \mathrm{CI} 0.22-1.01)$. Patients with a 1 -month follow-up recommendation were marginally more likely to be adherent than patients with a 6-month follow-up recommendation (OR=2.37, 95\% CI 0.96-5.85). Returning patients ( $\mathrm{OR}=1.63$, 95\% CI $0.89-2.98$ ) were marginally more likely to adhere to follow-up than new patients.

\section{Discussion}

In this study, unadjusted patient adherence rates of glaucoma follow-up appointments were similar between the CPEC clinic group (65.6\%), where patients received telephone reminders, and the glaucoma clinic group (68.1\%), where patients did not receive any telephone intervention. Although telephone reminders have not been found to improve 
Table 2 Characteristics of nonadherent and adherent patients

\begin{tabular}{|c|c|c|c|}
\hline Characteristic & Nonadherent, n (\%) & Adherent, n (\%) & $P$-value \\
\hline Service & & & 0.65 \\
\hline CPEC & $52(34.4)$ & $99(65.6)$ & \\
\hline Glaucoma & $46(31.9)$ & $98(68.1)$ & \\
\hline Age (years) & & & 0.038 \\
\hline$<50$ & $16(42.1)$ & $22(57.9)$ & \\
\hline $50-80$ & $59(28.6)$ & $\mid 47$ (7|.4) & \\
\hline$>80$ & $23(45.1)$ & $28(54.9)$ & \\
\hline Sex & & & 0.2 \\
\hline Female & $56(36.6)$ & $97(63.4)$ & \\
\hline Male & $42(29.6)$ & $100(70.4)$ & \\
\hline Race & & & 0.47 \\
\hline Black & $69(35.6)$ & $125(64.4)$ & \\
\hline White & $18(30.0)$ & $42(70.0)$ & \\
\hline Other & II (26.8) & $30(73.2)$ & \\
\hline Insurance type & & & 0.71 \\
\hline None & $6(28.6)$ & I5 (7I.4) & \\
\hline Medicaid & $12(26.7)$ & $33(73.3)$ & \\
\hline Medicare & $48(35.3)$ & $88(64.7)$ & \\
\hline Private & $32(34.4)$ & $61(65.6)$ & \\
\hline Insurance copay & & & 0.65 \\
\hline 0 & $56(31.6)$ & $12 \mid(68.4)$ & \\
\hline US\$1-\$20 & $12(40.0)$ & $18(60.0)$ & \\
\hline$>$ US $\$ 20$ & $30(34.1)$ & $58(65.9)$ & \\
\hline Glaucoma status & & & 0.84 \\
\hline Suspect & $51(33.8)$ & $100(66.2)$ & \\
\hline Definite & 47 (32.6) & $97(67.4)$ & \\
\hline Medications & & & 0.0005 \\
\hline$\leq 2$ & $85(38.8)$ & $134(6 \mid .2)$ & \\
\hline$>2$ & $13(17.1)$ & $63(82.9)$ & \\
\hline New patient & & & 0.25 \\
\hline No & $58(30.9)$ & $130(69.1)$ & \\
\hline Yes & $40(37.4)$ & $67(62.6)$ & \\
\hline \multicolumn{2}{|c|}{ Prior glaucoma surgery } & & 0.77 \\
\hline No & $80(33.6)$ & $158(66.4)$ & \\
\hline Yes & $18(31.6)$ & $39(68.4)$ & \\
\hline Visual acuity & & & 0.88 \\
\hline $20 / 20-20 / 100$ & $68(33.5)$ & $135(66.5)$ & \\
\hline$>20 / 100$ & $30(32.6)$ & $62(67.4)$ & \\
\hline IOP (mmHg) & & & 0.71 \\
\hline$<21$ & $78(33.8)$ & $153(66.2)$ & \\
\hline$\geq 21$ & $20(31.3)$ & $44(68.7)$ & \\
\hline CDR & & & 0.74 \\
\hline$<0.8$ & $51(34.0)$ & $99(66.0)$ & \\
\hline$\geq 0.8$ & $30(36.1)$ & $53(63.9)$ & \\
\hline VF MD & & & 0.32 \\
\hline$>-2$ & $7(18.4)$ & $31(81.6)$ & \\
\hline$\leq-2$ & $22(26.8)$ & $60(73.2)$ & \\
\hline Rec follow-up & & & 0.11 \\
\hline I-month & $36(27.1)$ & $97(72.9)$ & \\
\hline 3-month & $49(37.4)$ & $82(62.6)$ & \\
\hline 6-month & $13(4 \mid .9)$ & $18(58.1)$ & \\
\hline
\end{tabular}

Abbreviations: CDR, cup-to-disc ratio; CPEC, cataract and primary eye care; IOP, intraocular pressure; Rec, recommended; VF MD, visual field mean deviation.

glaucoma medication adherence,$^{18}$ most studies report that patients who receive a telephone or messaging reminder are more likely to keep their appointments. . $^{17,19,20}$

A possible explanation for the nearly similar adherence rates in this study were the differences in the baseline
Table 3 Odds ratios for multiple logistic regression analysis of risk factors for adherence with glaucoma follow-up visit

\begin{tabular}{llll}
\hline Risk factor & OR & $\mathbf{9 5 \% ~ C l ~}$ & P-value \\
\hline CPEC service vs glaucoma service & $\mathrm{I} .35$ & $0.79-2.32$ & 0.28 \\
Ocular medications $\leq 2$ vs $>2$ & 0.32 & $0.16-0.66$ & 0.0018 \\
Age $<50$ vs 50-80 years & 0.47 & $0.22-1.01$ & 0.02 \\
Age $>80$ vs 50-80 years & 0.45 & $0.23-0.87$ & 0.02 \\
I-month vs 6-month follow-up & 2.37 & $0.96-5.85$ & $0.06 \mathrm{I}$ \\
3-month vs 6-month follow-up & $\mathrm{I} .23$ & $0.54-2.84$ & $0.06 \mathrm{I}$ \\
Existing patient vs new patient & $\mathrm{I} .63$ & $0.89-2.98$ & $0.1 \mathrm{I}$ \\
\hline
\end{tabular}

Abbreviations: $\mathrm{Cl}$, confidence interval; $\mathrm{CPEC}$, cataract and primary eye care; $\mathrm{OR}$, odds ratio; vs, versus.

characteristics between the two groups: the group that received the phone intervention, the CPEC group, included patients who were mostly glaucoma suspects or at earlier stages of the disease, as opposed to the glaucoma clinic group, which included patients with more advanced glaucoma. It is well established that patients who understand the severity of their glaucoma are more likely to be adherent. ${ }^{5,12}$ For example, severe visual field loss, a family history of eye disease, or a diagnosis of blinding eye disease are factors that reduce patient dropout and nonadherence rates. ${ }^{12}$ Furthermore, patients with a definitive glaucoma diagnosis are more likely to return to the clinic compared to patients with a glaucoma suspect diagnosis. ${ }^{5}$ Thus, glaucoma clinic patients would be expected to have higher follow-up adherence rates than their counterparts in the CPEC group, though this difference may have been balanced by the telephone reminders. In fact, after adjustment for other factors, the OR for CEPC versus glaucoma was 1.35 indicating a possibility of some effect of telephone reminders on adherence, although the difference was not statistically significant.

In the Stubbs meta-analysis of various reminder methods, telephone reminders had the highest mean reduction of nonadherence, $9.4 \%$. Although they were demonstrated to be the most effective telecommunication tool in improving adherence, $8 \%$ of telephone studies did not show an improvement in adherence, and $40 \%$ did not demonstrate a statistically significant difference. ${ }^{17}$ Thus, the efficacy of telephone reminders varies based on the clinic and patient population under investigation. Other modalities such as text messaging and online open-access scheduling could also be investigated, but may be even more challenging to access for patients with vision impairments.

Patient demographics can also significantly affect patient satisfaction and patient adherence to follow-up. ${ }^{21}$ In comparing adherent and nonadherent subjects, we found that younger ( $<50$ years old) and older ( $>80$ years old) patients were marginally less likely to be adherent than those aged $50-80$ years. 
These differences may be due to younger subjects having less severe disease, possibly due to the early asymptomatic nature of glaucoma, and taking their disease less seriously; older subjects may have more barriers to care, including comorbidities, difficulties obtaining transportation for appointments, or problems navigating in the health care system. ${ }^{12}$ The number of patients with socioeconomic barriers to care, such as Medicaid insurance status or high copay amounts, were similar among adherent and nonadherent patients. ${ }^{11}$

Our study suggests that new patients may be less adherent than returning patients. Although this finding was not statistically significant in our study, it concurs with Bigger, ${ }^{22}$ who observed that patients who dropped out of care were likely to do so early on, but that adherence improved when patients received medical therapy. Even though there was a higher proportion of new patients in the CPEC clinic group that received telephone intervention, they had a similar adherence rate as the glaucoma clinic group.

Our strongest finding was that patients who were on more than two ocular medications were 3.11 times more likely to return for follow-up compared with patients on two or fewer ocular medications. Multiple medication use indicates either the need for lower target IOP or IOP more resistant to monotherapy and is therefore a proxy for more serious disease. Bigger $^{22}$ reported that medical therapy improved patient adherence. ${ }^{22}$ However, other authors have reported that patients on more than two ocular medications are more likely to be nonadherent. ${ }^{12}$ Patients who do not return to follow-up are also more likely to be nonadherent with medication dosing. ${ }^{5}$

Another proxy for disease severity was the patient's recommended time to follow-up. Patients with more severe eye disease are advised to come in more often. Thus, in our sample selection, we controlled for follow-up recommendations. We found there was a trend, although not statistically significant, where patients who were told to come in for 1-month follow-up were more likely to return than those scheduled for 6-month follow-up. It is possible that recommendation for relatively rapid return was associated with increased disease severity and therefore better adherence. However, it is also possible that shorter interval between visits facilitated adherence because it was easier for patients to pick an appointment time they would be likely to keep or it was easier for them to remember their scheduled appointment.

\section{Limitations}

There were several limitations of this study. First, the study was only powered to detect a difference of $15 \%$ or greater. A meta-analysis of the effect of telephone reminders found only a $9 \%$ improvement in patient adherence. ${ }^{17}$ Thus, this study may be underpowered.

Second, there were confounding factors that we were unable to eliminate in this study. The most significant potentially confounding factor was the patient population with more severe glaucoma in the glaucoma clinic. We attempted to control for differences in disease severity between the two groups by selecting a sample of patients with similar follow-up recommendations for each group. Increased disease severity and shorter follow-up intervals are known to be correlated with higher adherence, which may have limited our ability to detect adherence rate differences due to telephone reminders despite the multiple logistic regression model. As in all observational studies, there is potential for unmeasured confounding that could bias estimated effects.

Third, we relied on clinician coding to determine if patients had a definitive glaucoma diagnosis, as some patients did not have visual fields or CDR documented in their chart. Other authors have found this inconsistency in glaucoma care to be a common problem. ${ }^{11,23}$

Finally, the retrospective nature of this study resulted in significant limitations in interpretation of the results. Future prospective studies should have a more similar control group and include patient questionnaires to address patient perceptions into nonadherence with follow-up.

\section{Conclusion}

We found similar follow-up adherence rates between the CPEC clinic, which used telephone reminders, and the glaucoma clinic, which did not use any appointment reminders. We also found that patients older than age 80 , and patients with two or less ocular medications, were less likely to be adherent to follow-up eye appointment recommendations. Identifying patients at risk for nonadherence could allow targeted interventions, such as the use of patient navigators, to improve adherence, reduce costs related to vision loss, and prevent blindness.

\section{Acknowledgment}

Funding provided by the Glaucoma Service Foundation to Prevent Blindness, Philadelphia, PA, USA.

\section{Disclosure}

The authors report no conflicts of interest in this work.

\section{References}

1. Quigley HA, Broman AT. The number of people with glaucoma worldwide in 2010 and 2020. Br J Ophthalmol. 2006;90:262-267. 
2. Tham YC, Li X, Wong TY, Quigley HA, Aung T, Cheng CY. Global prevalence of glaucoma and projections of glaucoma burden through 2040: a systematic review and meta-analysis. Ophthalmology. 2014;121: 2081-2090.

3. Primary Open-Angle Glaucoma Summary Benchmarks for Preferred Practice Pattern ${ }^{\circledR}$ Guidelines; 2014. American Academy of Ophthalmology. The Eye MD Association. Available at: http://www.aao.org/asset. axd? $\mathrm{id}=$ dbbeb808-8bdd-474a-8253-68360f98e9e7. Accessed August 20, 2014.

4. DiMatteo MR. Variations in patients' adherence to medical recommendations: a quantitative review of 50 years of research. Med Care. 2004;42:200-209.

5. Kosoko O, Quigley HA, Vitale S, Enger C, Kerrigan L, Tielsch JM. Risk factors for noncompliance with glaucoma follow-up visits in a residents' eye clinic. Ophthalmology. 1998;105:2105-2111.

6. Olthoff CM, Schouten JS, van de Borne BW, Webers CA. Noncompliance with ocular hypotensive treatment in patients with glaucoma or ocular hypertension an evidence-based review. Ophthalmology. 2005;112:953-961.

7. Friedman DS, Hahn SR, Gelb L, et al. Doctor-patient communication, health-related beliefs, and adherence in glaucoma results from the Glaucoma Adherence and Persistency Study. Ophthalmology. 2008;115: 1320-1327.

8. Stewart WC, Chorak RP, Hunt HH, Sethuraman G. Factors associated with visual loss in patients with advanced glaucomatous changes in the optic nerve head. Am J Ophthalmol. 1993;116:176-181.

9. Norell SE. Monitoring compliance with pilocarpine therapy. Am J Ophthalmol. 1981;92:727-731.

10. Gurwitz JH, Yeomans SM, Glynn RJ, Lewis BE, Levin R, Avorn J. Patient noncompliance in the managed care setting. The case of medical therapy for glaucoma. Med Care. 1998;36:357-369.

11. Ngan R, Lam DL, Mudumbai RC, Chen PP. Risk factors for noncompliance with follow-up among normal-tension glaucoma suspects. Am J Ophthalmol. 2007;144:310-311.

12. Ashaye AO, Adeoye AO. Characteristics of patients who dropout from a glaucoma clinic. J Glaucoma. 2008;17:227-232.
13. Murakami Y, Lee BW, Duncan M, et al. Racial and ethnic disparities in adherence to glaucoma follow-up visits in a county hospital population. Arch Ophthalmol. 2011;129:872-878.

14. Brook RH, Fink A, Kosecoff J, et al. Educating physicians and treating patients in the ambulatory setting. Where are we going and how will we know when we arrive? Ann Intern Med. 1987;107:392-398.

15. Nguyen DL, Dejesus RS, Wieland ML. Missed appointments in resident continuity clinic: patient characteristics and health care outcomes. J Grad Med Educ. 2011;3:350-355.

16. Yancy WS Jr, Macpherson DS, Hanusa BH, et al. Patient satisfaction in resident and attending ambulatory care clinics. J Gen Intern Med. 2001;16:755-762.

17. Stubbs ND, Geraci SA, Stephenson PL, Jones DB, Sanders S. Methods to reduce outpatient non-attendance. Am J Med Sci. 2012;344:211-219.

18. Lim MC, Watnik MR, Imson KR, Porter SM, Granier AM. Adherence to glaucoma medication: the effect of interventions and association with personality type. J Glaucoma. 2013;22:439-446.

19. Guy R, Hocking J, Wand H, Stott S, Ali H, Kaldor J. How effective are short message service reminders at increasing clinic attendance? A metaanalysis and systematic review. Health Serv Res. 2012;47:614-632.

20. Bigna JJ, Noubiap JJ, Kouanfack C, Plottel CS, Koulla-Shiro S. Effect of mobile phone reminders on follow-up medical care of children exposed to or infected with HIV in Cameroon (MORE CARE): a multicentre, single-blind, factorial, randomised controlled trial. Lancet Infect Dis. 2014;14:600-608.

21. Fiebach NH, Wong JG. Taking care of patients in resident clinics: where do we stand? J Gen Intern Med. 2001;16:787-789.

22. Bigger JF. A comparison of patient compliance in treated vs untreated ocular hypertension. Trans Sect Ophthalmol Am Acad Ophthalmol Otolaryngol. 1976;81:277-285.

23. Hertzog LH, Albrecht KG, LaBree L, Lee PP. Glaucoma care and conformance with preferred practice patterns. Examination of the private, community-based ophthalmologist. Ophthalmology. 1996;103: 1009-1013.
Patient Preference and Adherence

\section{Publish your work in this journal}

Patient Preference and Adherence is an international, peer-reviewed, open access journal that focuses on the growing importance of patient preference and adherence throughout the therapeutic continuum. Patient satisfaction, acceptability, quality of life, compliance, persistence and their role in developing new therapeutic modalities and compounds to optimize

\section{Dovepress}

clinical outcomes for existing disease states are major areas of interest for the journal. This journal has been accepted for indexing on PubMed Central. The manuscript management system is completely online and includes a very quick and fair peer-review system, which is all easy to use. Visit http://www. dovepress.com/testimonials.php to read real quotes from published authors. 